\title{
Protocol for a Scoping Review of Research Practices in the Investigation of Bilingual Effects on Inhibition and Attentional Function in Young People
}

\author{
Adam John Privitera $^{1 *}$, Brendan Stuart Weekes ${ }^{1,2,3}$ \\ ${ }^{1}$ Faculty of Education, University of Hong Kong, Hong Kong SAR \\ ${ }^{2}$ Department of Psychology, University of Cambridge, Cambridge, UK \\ ${ }^{3}$ School of Psychology, University of Melbourne, Melbourne, Australia
}

\begin{abstract}
Background: While there is evidence in support of a bilingual advantage in executive function in children and adults, little work supports these effects in young people. This lack of support may result as consequence of a developmental ceiling effect on task performance in this age group. An alternative explanation can be found in the treatment of bilingualism as a categorical variable, and the use of exclusively fixed-effects methods of analysis. These methods treat bilinguals as a homogenous group, ignoring nontrivial differences between participants, and may contribute to this lack of evidence. This scoping review aims is to identify and summarize research practices in the investigation of bilingual effects on inhibition and attentional function in young people.
\end{abstract}

Methods/Design: The proposed scoping review will follow the five-stage framework proposed by Arksey and O'Malley (2005). Searches will be conducted across four databases using inclusive search strings. Study selection will follow the guidance of the PRISMA-ScR checklist. This review will include both published and unpublished work. A standardized data extraction spreadsheet will be used and data will be presented in tabular and graphic format in alignment with the objectives of the review.

Discussion: This review aims to provide a current understanding of research practices in the investigation of bilingual effects in young people as well as identify gaps in the literature. This review may also draw attention to methodological trends in the current literature that limit the conclusions researchers can draw.

Keywords: psycholinguistics, bilingualism, bilingual advantage, inhibition, attention, young people

\section{Background}

The majority of people on Earth are capable of communicating in more than one language (Grosjean, 2010). There is considerable debate around the topic of whether this bilingualism, which we define as proficiency in more than one language, confers non-linguistic benefits (Paap, 2019). Presently, there is evidence to support that speaking a second language provides a cognitive advantage in the form of improved executive function (Antoniou, 2019). Because executive function, which is thought to operate through attentional functions in a manner that

* Correspondence to Adam John Privitera. Email: aprivite@qq.com

Social Science Protocols, November 2021, 1-7.

http://dx.doi.org/10.7565/ssp.2021.5760 
is goal directed (Braver, 2012), is proposed to consist of multiple, separable abilities (Miyake et al., 2000), separate tasks are often used in the assessment of differences in updating, shifting, or inhibition. One of these abilities, inhibition or inhibitory control, is considered a likely candidate for a bilingual effect on executive function to manifest. This hypothesis stems from the observation that bilinguals must regularly inhibit an active but unneeded language when communicating in a bilingual context (Green, 1998).

Previous investigations of bilingual effects on executive function have produced mixed findings. Studies reporting improved task performance of bilinguals compared to monolinguals cite improved performance on incongruent trials (i.e., trials presenting conflict) as evidence in support of a bilingual inhibitory control advantage. Evidence supporting a more general effect of bilingualism on executive function, the bilingual executive processing advantage, is more consistently reported, and presents as improved performance across all trial conditions (Hilchey \& Klein, 2011). This advantage, thought to result from the increased monitoring demands of bilingual environments, aligns more with a bilingual advantage in attention than on inhibition, and is influenced by task monitoring demands (Costa et al., 2009). However, evidence for a bilingual advantage, if observed at all, is considered to be artefactual and a result of design flaws, specific task conditions, publication bias, or other non-linguistic factors (De Bruin \& Della Sala, 2019; Lowe et al., 2021; Paap, 2019; Paap et al., 2015; Ware et al., 2020).

Developmentally, executive function is thought to peak during the middle teens and early 20 s in young people (Anderson, 2002). The proposed scoping review will adopt the World Health Organization's (WHO) definition of young people as those persons in the period of development occurring between the ages of 10-24, which includes adolescence. As it follows, a bilingual effect on executive function, including inhibition, may be difficult to measure in these samples as a consequence of a ceiling effect on task performance (Bialystok, 2016). While there is evidence that improvement in task performance after multiple testing sessions can be measured during this developmental peak in function (Paap et al., 2014), researchers may have neglected investigating bilingual effects in young people.

Despite the heterogeneity and multidimensionality of bilingual experience, (Gullifer et al., 2021), studies investigating bilingual effects often classify bilingualism as a categorical variable, assigning participants based on artificial and oversimplified criteria (Luk \& Bialystok, 2013). This practice is not only artefactual and biased methodologically, it lacks ecological validity, ignoring non-trivial differences in bilingual experience (De Bruin, 2019). Furthermore, this classification does not consider linguistic similarity between utilized languages, a variable which may influence the emergence of bilingual effects (Coderre \& Van Heuven, 2014; Kuzmina et al., 2019; Weekes, 2020). The impact of these methodological decisions is further exacerbated through the use of fixed-effects analyses which prevent full consideration of individual differences. Recently, movement toward the adoption of mixedeffects analyses by researchers in psycholinguistics (Linck \& Cunnings, 2015), in combination with the collection of detailed language history data (Li et al., 2020; Marian et al., 2007) has helped to ameliorate the issues associated with previous analysis methods.

The main objective of the proposed scoping review is to identify and summarize research practices in the investigation of bilingual effects on inhibition and attentional function in young people. The motivation for this scoping review is driven, in part, by the need to map out research on a potentially underrepresented age group with the goal of better informing future investigation. Mapping out where this research is conducted, the diversity of language combinations included in bilingual samples, and the methods used to analyse these data further supports that goal, and also serves to highlight methodological decisions that may undermine the strength of conclusions that can be drawn regarding the influence of bilingualism on inhibition and attentional function. Additionally, we aim to identify gaps in the literature that merit further investigation by future research.

Social Science Protocols, November 2021, 1-7.

http://dx.doi.org/10.7565/ssp.2021.5760 


\section{Methods/Design}

\subsection{Scoping review methodological framework}

A scoping review of research assessing the presence of a bilingual advantage in cognitive functioning in young people will conducted. Based on the aims of the proposed review (i.e., identify existing research and gaps in the literature), the scoping review method was determined to be the most appropriate (Munn et al., 2018). The five-stage methodological framework proposed by Arksey and O'Malley (2005) will guide the conduct of the proposed scoping review. These stages are: (1) identifying the research question; (2) identifying relevant studies; (3) study selection; (4) charting the data; and (5) collating, summarizing, and reporting the results. Critical appraisal of identified research will not be conducted as the proposed review aims to identify existing research and gaps in the literature, and is not concerned with whether that research is of high quality. This protocol has been preregistered with the Centre for Open Science (DOI 10.17605/OSF.IO/8HQDY).

\subsection{Identifying the research question}

The primary research question of the proposed scoping review is "what are the current research practices in the investigation of bilingual effects on inhibition and attentional function in young people?". The research sub-questions are:

1. What tasks are used to assess bilingual effects on inhibition?

2. What tasks are used to assess bilingual effects on attentional function?

3. Where are studies on bilingual effects conducted?

4. What language combinations have been reported in bilingual samples?

5. Which analysis methods are used in the analysis of these data?

\subsection{Identifying relevant studies}

Searches will be conducted using the following online databases: PubMed, ProQuest, Scopus, and PsyArXiv. All studies published prior to July, 2021 were considered for inclusion in the review. The decision to include both peer-reviewed and grey literature was made to help ensure that the most comprehensive collection of research available was considered for inclusion. Search strings will be customized based on the conventions of each online database, and will include combinations of the following keywords: "bilingual*", "multilingual*", "child*", "adolescen*", "young adult*", "executive", "attention*", and "inhibit*". The inclusion of the "*" character in a search string keyword allows for searches to consider keywords that are sometimes used interchangeably with our keywords of interest (e.g., "bilingual*" would return articles that included the keywords "bilingual", "bilinguals", and "bilingualism"). Search fields were limited to article titles, abstracts, and keywords. Search strings will be piloted to ensure that known relevant papers are returned across the four databases used. Results from all databases will be combined and duplicates within and across databases will be identified and removed prior to screening for inclusion.

\subsection{Selection of studies}

The selection of studies for inclusion in the proposed review will follow the guidance of the Preferred Reporting Items for Systematic Reviews and Meta-Analyses Extension for Scoping Reviews (PRISMA-ScR) checklist (Tricco et al., 2018). The process of study selection will be documented in the form of a PRISMA flow diagram (Page et al., 2021). Studies will first be screened through reading of the title and abstract. Those studies identified as candidates for inclusion will then go through full-text screening. The following eligibility criteria will be applied when determining if a study is to be included in the review:

Social Science Protocols, November 2021, 1-7.

http://dx.doi.org/10.7565/ssp.2021.5760 
Inclusion criteria. Studies meeting the following criteria will be included in the proposed review:

- Conducted using a sample of bilinguals with an average age between 10-24 years.

- Include at least one task assessing inhibition or attentional function.

- Published prior to July, 2021

- Published in English

Exclusion criteria. Studies meeting the following criteria will be excluded from the proposed review:

- Focused on other aspects of executive control

- Only available as conference abstract

- Full text is unavailable

All attempts will be made in order to include potentially relevant studies in the proposed review. This includes but is not limited to contacting corresponding authors of original studies in order to (1) obtain full texts of articles or (2) seek additional information in order to determine whether an article meets the inclusion criteria. The search and selection processes are expected to be completed by July, 2021.

\subsection{Charting the data}

Data will be extracted using a digital spreadsheet-based table customized to the needs of the proposed review (Table 1). When possible, the entry of data will be handled through autocomplete of previously entered responses in order to prevent errors during entry. Extracted data will include: (1) study authors; (2) year of publication; (3) country in which study was conducted; (4) average age of participants in the bilingual sample; (5) language combination used by participants in the bilingual sample; (6) task(s) used to assess inhibition; (7) task(s) used to assess attentional function; and, (8) method used for data analysis. Finalization of the extraction table is expected to be completed by the end of July, 2021.

\subsection{Collating, summarizing, and reporting the results}

Data from included studies will be summarized in both tabular and narrative report format. Table format will be identical to the extraction table described above. Narrative report results will be summarized in relation to each of the research questions as well as the overall focus of the proposed review. The identification of gaps will focus on geographic areas where research on this topic is not being conducted, language combinations that are not regularly found in bilingual samples used, analysis methods that are not aligned with emerging best practices in this field, as well as general methodological considerations in need of further exploration. Full results of the proposed review will be reported in the form of a full-length manuscript that will be submitted for publication in a peer-review journal.

\section{Discussion}

The proposed scoping review aims to provide a current understanding of the research practices used in the investigation of bilingual effects on inhibition and attentional function in young people as well as identify gaps in the literature in need of further exploration. We expect that this review could provide researchers in a wide range of fields with a better understanding of how bilingual effects are studied in this age group. This review may also draw attention to methodological trends in the current literature that limit the conclusions researchers can draw about the impact of bilingualism on inhibition and attentional function.

Social Science Protocols, November 2021, 1-7. 
Possible limitations of the proposed review include the narrow age range outlined in the inclusion criteria. The findings from this review are likely not applicable to age groups outside of young people. Focusing on only inhibition and attentional function will likely limit the application of our findings to only research on specific domains of executive function. Additionally, because we do not plan to conduct critical appraisal on included studies, there may be issues related to the quality of research summarized in the review.

\section{Table 1}

Data extraction spreadsheet with example entries in top row

\begin{tabular}{|c|c|c|c|c|c|c|c|c}
\hline Study & Source & Country & Sample & AVG Age & Language Comb. & Inhibition Task(s) & Attention Task(s) & Analysis \\
\hline Author et al., 2019 & Article & Qatar & $\begin{array}{c}\text { BL: } 100 ; 13 \mathrm{M} \\
\text { ML: } 100 ; 15 \mathrm{M}\end{array}$ & $\begin{array}{c}\text { BL: 22 } \\
\text { ML: 22 }\end{array}$ & English-Arabic & Simon & ANT & ANOVA \\
\hline & & & & & & & & \\
\hline \\
\hline
\end{tabular}

\section{Declarations}

Availability of data and material: All data associated with the proposed review will be included in the final published version. Data will also be uploaded to Open Science Framework (OSF).

Competing interests: The authors declare that they have no competing interests.

Funding: No external funding.

Authors' contributions: Adam John Privitera: conceptualization, methodology, writingoriginal draft, writing-review and editing. Brendan Stuart Weekes: writing-review and editing.

\section{References}

Anderson, P. (2002). Assessment and development of executive function (EF) during childhood. Child Neuropsychology, 8(2), 71-82.

Antoniou, M. (2019). The advantages of bilingualism debate. Annual Review of Linguistics, $5,395-415$.

Arksey, H., \& O'Malley, L. (2005). Scoping studies: towards a methodological framework. International Journal of Social Research Methodology, 8(1), 19-32.

Social Science Protocols, November 2021, 1-7. 
Bialystok, E. (2016). The signal and the noise: Finding the pattern in human behavior. Linguistic Approaches to Bilingualism, 6(5), 517-534.

Braver, T. S. (2012). The variable nature of cognitive control: a dual mechanisms framework. Trends in Cognitive Sciences, 16(2), 106-113.

Coderre, E. L., \& Van Heuven, W. J. B. (2014). The effect of script similarity on executive control in bilinguals. Frontiers in Psychology, 5, 1070.

Costa, A., Hernández, M., Costa-Faidella, J., \& Sebastián-Gallés, N. (2009). On the bilingual advantage in conflict processing: Now you see it, now you don't. Cognition, 113(2), 135149.

De Bruin, A. (2019). Not all bilinguals are the same: A call for more detailed assessments and descriptions of bilingual experiences. Behavioral Sciences, 9(3), 33.

De Bruin, A., \& Della Sala, S. (2019). The bilingual advantage debate: Publication biases and the decline effect. In J. W. Schwieter (Ed.), The Handbook of the Neuroscience of Multilingualism. Wiley.

Green, D. W. (1998). Mental control of the bilingual lexico-semantic system. Bilingualism: Language and Cognition, 1(2), 67-81.

Grosjean, F. (2010). Bilingual. Harvard university press.

Gullifer, J. W., Kousaie, S., Gilbert, A. C., Grant, A., Giroud, N., Coulter, K., Klein, D., Baum, S., Phillips, N., \& Titone, D. (2021). Bilingual language experience as a multidimensional spectrum: Associations with objective and subjective language proficiency. Applied Psycholinguistics, 42, 1-34.

Hilchey, M. D., \& Klein, R. M. (2011). Are there bilingual advantages on nonlinguistic interference tasks? Implications for the plasticity of executive control processes. Psychonomic Bulletin \& Review, 18(4), 625-658.

Kuzmina, E., Goral, M., Norvik, M., \& Weekes, B. S. (2019). What influences language impairment in bilingual aphasia? A meta-analytic review. Frontiers in Psychology, 10, 445.

Li, P., Zhang, F., Yu, A., \& Zhao, X. (2020). Language History Questionnaire (LHQ3): An enhanced tool for assessing multilingual experience. Bilingualism: Language and Cognition, 23(5), 938-944.

Linck, J. A., \& Cunnings, I. (2015). The utility and application of mixed-effects models in second language research. Language Learning, 65(S1), 185-207.

Lowe, C. J., Cho, I., Goldsmith, S. F., \& Morton, J. B. (2021). The Bilingual Advantage in Children's Executive Functioning Is Not Related to Language Status: A Meta-Analytic Review. Psychological Science, 0956797621993108.

Luk, G., \& Bialystok, E. (2013). Bilingualism is not a categorical variable: Interaction between language proficiency and usage. Journal of Cognitive Psychology, 25(5), 605621.

Marian, V., Blumenfeld, H. K., \& Kaushanskaya, M. (2007). The Language Experience and Proficiency Questionnaire (LEAP-Q): Assessing language profiles in bilinguals and multilinguals. Journal of Speech, Language, and Hearing Research.

Miyake, A., Friedman, N. P., Emerson, M. J., Witzki, A. H., Howerter, A., \& Wager, T. D. (2000). The unity and diversity of executive functions and their contributions to complex "frontal lobe" tasks: A latent variable analysis. Cognitive Psychology, 41(1), 49-100.

Munn, Z., Peters, M. D. J., Stern, C., Tufanaru, C., McArthur, A., \& Aromataris, E. (2018). Systematic review or scoping review? Guidance for authors when choosing between a systematic or scoping review approach. BMC Medical Research Methodology, 18(1), 1-7. Paap, K. (2019). The bilingual advantage debate: Quantity and quality of the evidence. The Handbook of the Neuroscience of Multilingualism, 701-735.

Social Science Protocols, November 2021, 1-7.

http://dx.doi.org/10.7565/ssp.2021.5760 
Paap, K. R., Johnson, H. A., \& Sawi, O. (2015). Bilingual advantages in executive functioning either do not exist or are restricted to very specific and undetermined circumstances. Cortex, 69, 265-278.

Paap, K. R., Wagner, S., Johnson, H., Bockelman, M., Cushing, D., \& Sawi, O. (2014). 20,000 Flanker trials: are the effects reliable, robust, and stable? Poster Presented at the Association for Psychological Science, San Francisco, CA.

Page, M. J., Moher, D., Bossuyt, P. M., Boutron, I., Hoffmann, T. C., Mulrow, C. D., Shamseer, L., Tetzlaff, J. M., Akl, E. A., \& Brennan, S. E. (2021). PRISMA 2020 explanation and elaboration: updated guidance and exemplars for reporting systematic reviews. $B M J, 372$.

Tricco, A. C., Lillie, E., Zarin, W., O'Brien, K. K., Colquhoun, H., Levac, D., Moher, D., Peters, M. D. J., Horsley, T., \& Weeks, L. (2018). PRISMA extension for scoping reviews (PRISMA-ScR): checklist and explanation. Annals of Internal Medicine, 169(7), 467-473.

Ware, A. T., Kirkovski, M., \& Lum, J. A. G. (2020). Meta-analysis reveals a bilingual advantage that is dependent on task and age. Frontiers in Psychology, 11(1458).

Weekes, B. S. H. (2020). Literacy in Contact and in Context. Letrônica, 13(4), e37538e37538. 